\title{
The need for trainee-led surgical research collaborative in Sri Lanka
}

\author{
Umesh Jayarajah, Oshan Basnayake \\ Postgraduate Institute of Medicine, University of Colombo, Sri Lanka
}

Keywords: Research collaborative; trainee-led research; surgical research

\section{Introduction}

The value of medical research in the undergraduate and postgraduate medical curriculum is increasingly recognised [1]. Many professional medical bodies promote undergraduate and post-graduate trainees to gain some experience in research, not only to assist in carrying out original studies but also to cultivate skills to become accomplished research consumers. This will enable them to be informed about current evidence and enhance their professional practice [1].

However, there have been many obstacles to fulfil this aim. Post-graduate trainees are occupied with their clinical practice, especially in a field like surgery where necessary practical skills must be learned in addition to the core clinical knowledge [1]. Furthermore, partly due to inadequate guidance, many trainees feel discouraged about research altogether or commence impractical or over-ambitious studies often ending up in failures discouraging further attempts in research. Outputs from such attempts are unlikely to contribute to science and are often not disseminated. This is because of too small sample size, lack of novelty, singlecentre, lack of proper follow-up and lack generalizability [2]. Furthermore, there is a general lack of formal training in research methodology.

In general, there are several disadvantages related to small scale single centre studies. Underpowered studies with a small sample size and broad confidence intervals result in findings that contribute minimally to science and unlikely to change clinical practice [3]. Many such studies failed to get published in peer-reviewed journals. However, there is an increasing trend to conduct scientific work by collaborative networks [4]. Such collaborative networks enable a group of geographically scattered researchers of common interests to communicate and contribute towards a common goal. This is

Correspondence: Umesh Jayarajah

E-mail: umeshe.jaya@gmail.com

(D)https://orcid.org/0000-0002-0398-5197

Received: 14-11-2020 Accepted: 09-03-2021

DOI: http://doi.org/10.4038/sljs.v39i1.8753 feasibly achieved through online platforms using modern information technology [5].

Several difficulties about working across geographical distance have been identified. These include issues related to ownership, authorship, funding, intellectual property, trust and communication [1]. The establishment of well organised collaborative networks with well documented and transparent protocols have been helpful to overcome these shortcomings [1]. A functional model for a collaborative research network is shown in figure 1 .

In the UK, over the past decade, several trainee-led regional collaborative in general surgery and other specialities have been established [1]. This approach enables to include a sufficiently large sample in a short duration and prevents duplication of data. Moreover, this enables to conduct of multicentre pragmatic studies and allows for greater generalizability in contrast to single centre short term studies [6]. Trainees follow clinical rotation in multiple hospitals nationwide and are in regular contact with each other and therefore, are ideal to implement this model. As trainees become independent practitioners, a culture of surgical research and trials could be distilled in the country's surgical practice [6]. So far, several surgical research collaborative networks have been initiated in the UK with considerable success with large scale trainee-driven cohort studies and clinical trials [6].

There is increasing evidence that research experiences of trainee doctors have long term benefits in their subsequent careers and may also enhance the opportunities for overseas training [7]. This may positively influence lifelong academic inquisitiveness and contributions to teaching, ultimately improving patient care. Due to the above reasons, a research forum led by trainees with the support and guidance of experienced researchers will be useful. Such an initiative should allow all trainees within a region or country to team up irrespective of the geographical location, academic schedule and long term academic interest. In conclusion, a trainer-led collaborative platform may be an effective model to enhance and promote high-quality surgical research in Sri Lanka. 


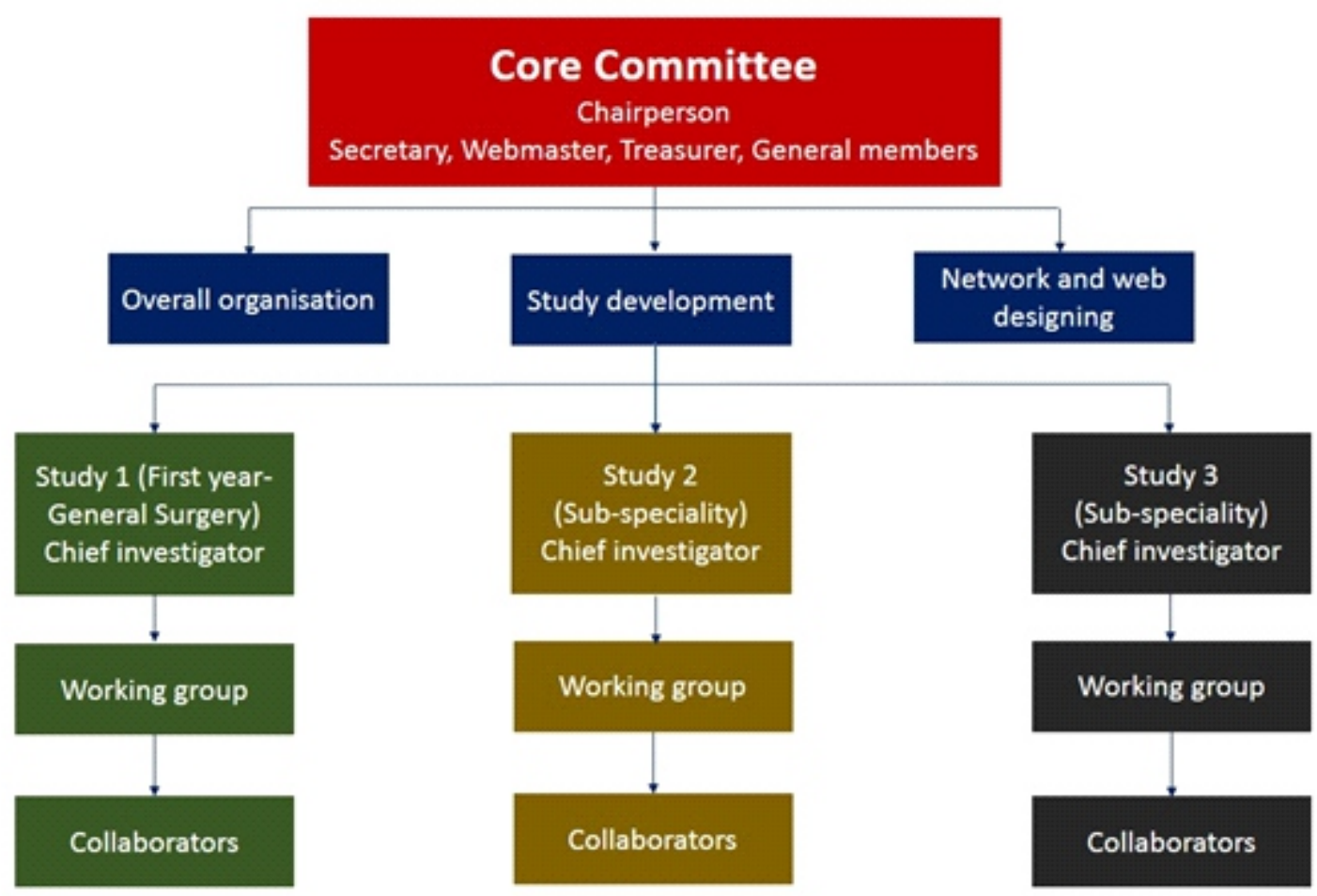

Figure 1. Functional model for a collaborative research network

\begin{abstract}
All authors disclose no conflict of interest. The study was conducted in accordance with the ethical standards of the relevant institutional or national ethics committee and the Helsinki Declaration of 1975, as revised in 2000 .
\end{abstract}

\section{References}

1. Dowswell G, Bartlett D C, Futaba K, Whisker L, Pinkney T D. How to set up and manage a trainee-led research collaborative. BMC Medical Education 2014; 14: 94.

https://doi.org/10.1186/1472-6920-14-94

2. Brubaker L, Kenton K. Clinical research education study teams: a research curriculum for obstetric and gynecology residents. Obstetrics \& Gynecology 2011; 117: 1403-7. https://doi.org/10.1097/AOG.0b013e3182179705

3. Bhandari M, Sprague S, Schemitsch EH. Resolving controversies in hip fracture care: the need for large collaborative trials in hip fractures. Journal of orthopaedic trauma 2009; 23: 479. https://doi.org/10.1097/BOT.0b013e3181a772e3
4. Newman ME. The structure of scientific collaboration networks. Proceedings of the national academy of sciences 2001; 98: 404-9. https://doi.org/10.1073/pnas.98.2.404

5. Bos N, Zimmerman A, Olson J et al. From shared databases to communities of practice: A taxonomy of collaboratories. Journal of Computer-Mediated Communication 2007; 12: 652-72. https://doi.org/10.1111/j.1083-6101.2007.00343.x

6. Bhangu A, Kolias AG, Pinkney T, Hall NJ, Fitzgerald JE. Surgical research collaboratives in the UK. The Lancet 2013; 382: 1091-2. https://doi.org/10.1016/S0140-6736(13)62013-9.

7. Bhattacharya SD, Williams JB, de la Fuente SG, Kuo PC, Seigler HF. Does protected research time during general surgery training contribute to graduates' career choice? The American surgeon 2011; 77:907-10.

https:/www.ncbi.nlm.nih.gov/pmc/articles/PMC3720679/

5 